\title{
Erk activation as a possible mechanism of transformation of subependymal nodule into subependymal giant cell astrocytoma
}

\author{
Monika Siedlecka ${ }^{1}$, Stanislaw Szlufik ${ }^{1}$, Wieslawa Grajkowska ${ }^{2,3}$, Marcin Roszkowski ${ }^{4}$, Jarosław Jóźwiak ${ }^{1}$ \\ ${ }^{1}$ Department of Histology and Embryology, Medical University of Warsaw, ${ }^{2}$ Department of Clinical and Experimental Neuropathology, \\ Mossakowski Medical Research Centre, Polish Academy of Sciences, Warsaw, ${ }^{3}$ Department of Pathology, The Children's Memorial \\ Health Institute, Warsaw, ${ }^{4}$ Department of Neurosurgery, The Children's Memorial Health Institute, Warsaw, Poland
}

\begin{abstract}
Introduction: Subependymal nodule (SEN) and subependymal giant cell astrocytoma (SEGA) are brain lesions frequently found in tuberous sclerosis (TS). As about 10-15\% of SENs enlarge and transform into SEGAs, we examined here the possible mechanism of the phenomenon.

Material and methods: Using Western blot we studied 1 SEN and 3 SEGA samples; SEN and 1 SEGA came from the same TS patient. We evaluated e.g. the activation of the phosphorylated forms of proteins belonging to Akt, Erk and mTOR pathways.

Results: Differences in Erk pathway activation between SEN and SEGA were found. There was no upregulation of $p$-Erk, $p$-Mek or $p$-RSK 1 in the SEN specimen, whilst we found these proteins to be significantly uptriggered in SEGA samples. Also, for the first time, we found $p-A k t, p-G S K 3 \beta$ and $p$-PDK 1 upregulated in both SEN and SEGA from the same TS patient.

Conclusions: Our current study shows for the first time the possible mechanism of SEN/SEGA transformation, where Erk pathway hyperactivation seems to be significant. We hypothesize that SEN/SEGA transformation may depend on Erk potentiation.
\end{abstract}

Key words: subependymal giant cell astrocytoma, subependymal nodule, Erk, tuberous sclerosis.

\section{Introduction}

Tuberous sclerosis (TS) is a neurological autosomal dominant disorder, characterized by high variability of clinical presentation, as well as high penetrance, leading to an incidence estimated as $1: 6000$ [11]. Tuberous sclerosis may be associated with multiple hamartomas and neoplasms in several organs, mainly in the skin, brain, heart, kidneys and lungs $[5,15]$. The multisystem, usually benign, non-invasive lesions vary in size and number and are randomly distributed throughout the body. Thus, clinical manifestations can vary widely, even among close relatives. Major and minor clinical criteria serve to establish the diagnosis [15]. Recently, also genetic diagnostic criteria were postulated [17].

Brain-related manifestations are associated with the highest morbidity in TS patients. About $85 \%$ of patients suffer from neurological complications, such as epilepsy, intellectual disability or autism. Epilepsy 
is very often drug-resistant and has an early onset; seizures start during the first year of life in most patients [5]. Among neuropathological features of TS, the most frequent is the presence of cortical and subcortical tubers, a type of cortical dysplasia found in $90 \%$ of patients; subependymal nodules (SENs) are found in about $80 \%$ of TS patients and subependymal giant cell astrocytomas (SEGA; called subependymal giant cell tumors by some authors) occur in about $5-10 \%$ of TS patients.

Subependymal nodules are usually small, asymptomatic hamartomas, located in the lateral ventricles or adjacent to the caudate nucleus. Subependymal nodules usually develop during fetal life, and commonly calcify. The lesions can occur as single, irregular nodules, not exceeding $1 \mathrm{~cm}$ in diameter, or multiple lesions (forming "candle guttering"). On magnetic resonance imaging (MRI) scans, SENs are hyperintense on T1-weighted images, especially in neonates, whilst on T2-weighted images, because of their calcification, they exhibit an iso- or hypointense signal. Also variable enhancement after contrast injection is commonly seen, but this phenomenon is not considered to be of prognostic value [12]. In about $10-15 \%$ of cases SENs enlarge gradually and transform into SEGAs. This process is the fastest during the first 2 decades of life: SEGAs are rarely found in newborns, but their prevalence increases with age [5]. Histologically, the nodules consist of spindle glial cells and large cells, similar to giant cells in cortical tubers, positive for GFAP and sometimes for neuronal markers [10].

Subependymal giant cell tumors are low-grade (WHO grade 1) glial tumors, highly vascularised and located typically near the foramen of Monro, at the caudothalamic groove. The tumors consist of spindle cells, gemistocytic-like cells and giant cells. Immunohistochemical analysis reveals mainly glial, but also neuronal features. The dysmorphic glial cells in SEGA can form pseudorosettes or clusters. The giant cells are similar to those in cortical tubers. Also some foci of necrosis can be present, as well as some mitotic figures; the labeling index of $\mathrm{Ki}-67$ is about $1 \%[1,2,4]$, although rare cases of higher mitotic index are also reported [3].

The diagnostic definition of SEGA includes caudothalamic groove lesions which exceed $1 \mathrm{~cm}$ in diameter or subependymal lesions growing consecutively, irrespective of the tumor size [15]. On CT, SEGAs are iso- or hyperattenuated and commonly calcified.
On MRI they have mixed signal intensity and reveal strong enhancement after contrast administration [16]. Even though SEGAs grow slowly, eventually, because of their significant size, they can cause cerebrospinal flow obstruction, increase of intracranial pressure and hydrocephalus [10].

The clinical presentation results from mutation in the suppressor gene TSC1 (tuberous sclerosis complex 1) or TSC2 (tuberous sclerosis complex 2), encoding hamartin and tuberin, proteins forming a heterodimer. The hamartin-tuberin heterodimer acts in normal conditions as the suppressor of the mammalian target of rapamycin (mTOR), a serine-threonine kinase known as the central regulator of cellular growth, proliferation and protein synthesis. Mammalian target of rapamycin receives an input from various signals, such as growth factor stimulation, nutrient availability or the state of oxygenation. In TS, as the TSC1/TSC2 suppressor complex is nonfunctional, the upstream kinase cascades, such as protein kinase B (Akt) and extracellular signal-regulated kinase (Erk) pathways, are capable of mTOR upregulation through the phosphorylation of cell-cycle regulators as well as transcription factors [7].

In this study, we tried to determine why SENs start to grow and transform into SEGAs. First of all, we focused on mTOR kinase and its activators. It is already known that both Akt and Erk pathways can be upregulated in SEGAs [6,7]. However, we do not know any previous study on mTOR, Akt or Erk pathways on SEN specimens. For this reason, in the current study we examined for the first time the activation of the proteins belonging to the above-mentioned pathways in SEN and compared the results with the protein activation pattern in SEGAs.

\section{Material and methods}

\section{Tissue samples}

All studied SEN and SEGA samples, as well as control tissues, were retrieved from the Department of Pathology, Children's Memorial Hospital in Warsaw, Poland. Three studied SEGA samples and one SEN specimen from patients with TS complex, were classified by the neuropathologists, according to international criteria $[14,15]$. One of the SEGA samples came from the same TS patient from whom we obtained the SEN sample. As a positive control we used medulloblastoma, where Akt and Erk pathway activation was documented [17]. 


\section{Sample preparation}

Our research was performed by the Western blot method. All the lysates were prepared simultaneously, in order to avoid differences in conditions during sample processing. Tissues prepared for electrophoresis were homogenized in a tissue grinder with RIPA lysis buffer $(20 \mathrm{mM}$ Tris $\mathrm{pH} 7.5,150 \mathrm{mM} \mathrm{NaCl}$, $1 \%$ Nonident P-40, 0.5\% sodium deoxycholate, $1 \mathrm{mM}$ EDTA, $0.1 \%$ SDS) with $50 \mathrm{mM}$ sodium fluoride and $1 \mathrm{mM}$ sodium orthovanadate, supplemented with 1x Complete Protease Inhibitor (Roche, Indianapolis, IN) and Phosphatase Inhibitor Cocktail I (Sigma-Aldrich, St. Louis, MO). The storage temperature of prepared lysates was $-80^{\circ} \mathrm{C}$.

\section{Western blot}

Thawed tissue lysates were diluted in Bio-Rad's Laemmli Sample Buffer (62.5 mM Tris- $\mathrm{HCl}$, pH 6.8;
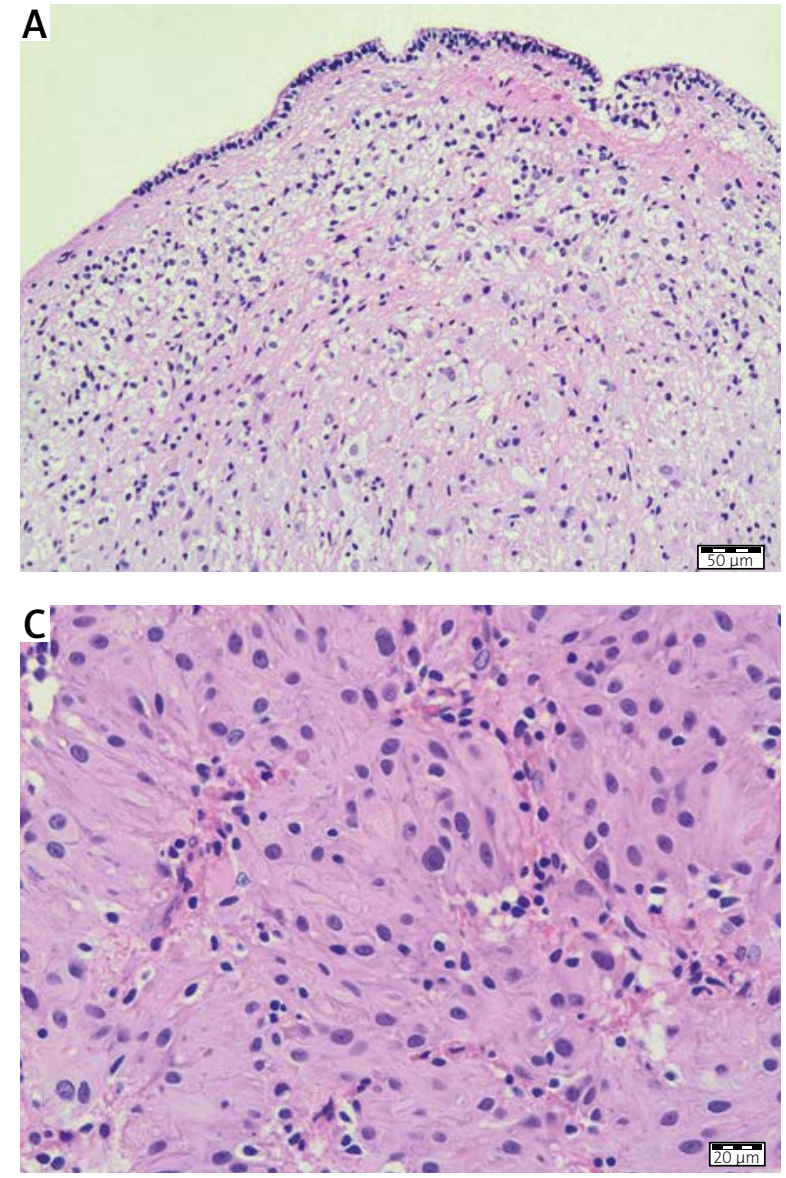

25\% glycerol; 2\% SDS; 0.01\% Bromophenol Blue; B-mercaptoethanol for a final concentration of $5 \%$ was added) in a 1: 1 ratio. Subsequently the samples were heated for 5 minutes at $95^{\circ} \mathrm{C}$. About $20 \mu \mathrm{g}$ of protein tissue extract was put into each well of $10 \%$ polyacrylamide gel and subjected to SDS PAGE.

After completing SDS PAGE and electrophoretic transfer onto PVDF membrane, the blots were blocked with $5 \%$ non-fat dry milk in TBST (Tris buffered saline, $0.05 \%$ Tween). Afterwards, the membranes were incubated with the primary antibody (at $4^{\circ} \mathrm{C}$, for 16 hours) and respective HRP-conjugated secondary antibody (for 30 minutes at room temperature; diluted with $2.5 \%$ non-fat dry milk in TBST). We used West Pico chemiluminescence substrate (Pierce, Rockford, IL) for protein detection. Equal protein loading was evaluated by Ponceau $\mathrm{S}$ and $\alpha$-tubulin staining.
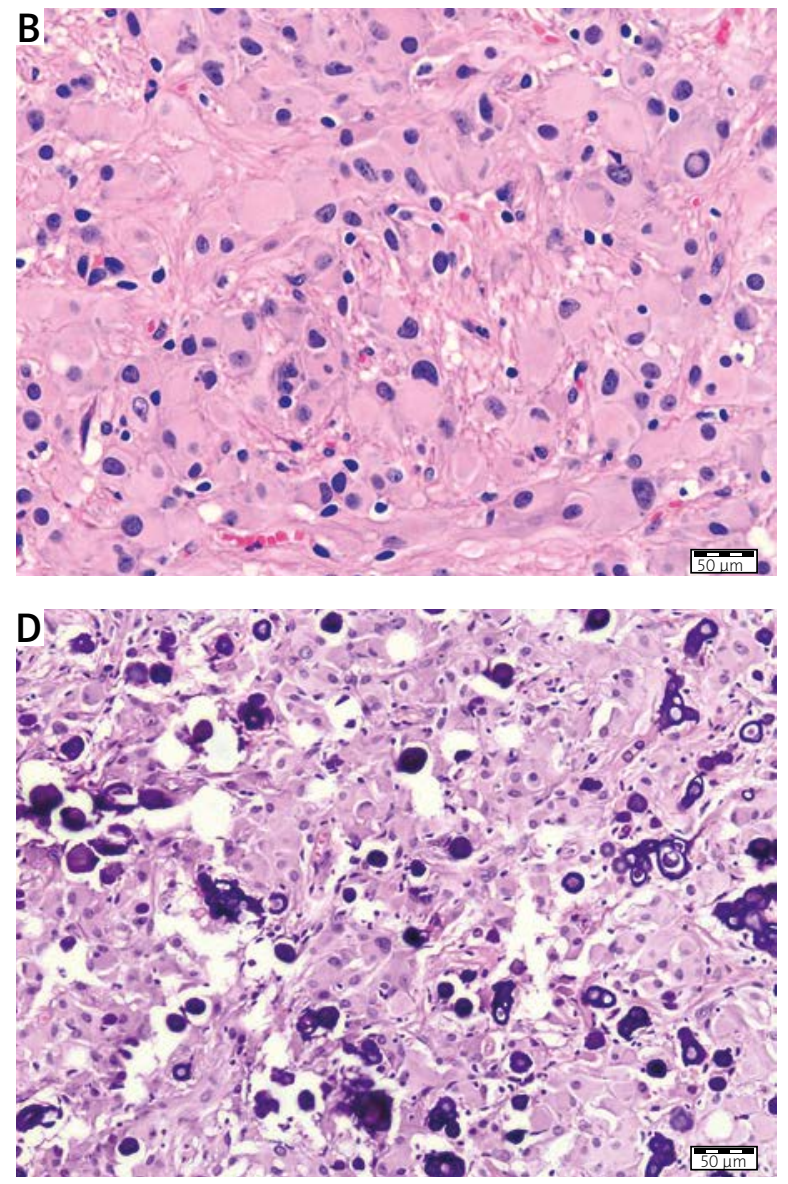

Fig. 1. A) Subependymal nodule covered by ependyma with largest cells in the central part of the lesion. B) Subependymal nodule showing large astrocytic cells with glassy cytoplasm. C) Subependymal nodule displaying spindle cell aggregate around blood vessels. D) Subependymal nodule with numerous calcifications. H\&E staining. 


\section{Antibodies for Western blot}

The following primary antibodies were purchased from Cell Signaling Technologies: PathScan PDGFR Activity Assay Multiplex Western Detection Cocktail; p-Akt/PKB Ser 473, p-GSK3 $\beta$ Ser 9, p-PDK1 Ser 24, p-S6rp Ser 235/236; p-MEK 1/2 Ser 221, p-p90-RSK Ser 380; p-Erk Thr 202/Tyr 204; total-Erk; Rheb; Raptor; elF4E; p-SAPK/JNK Thr183/Tyr185.

The following primary antibodies were purchased from Santa Cruz Biotechnology: alpha-tubulin; cyclin D1; Bax; Bad; caspase 9.

\section{Results}

\section{Neuropathological features}

Histopathological features of both SEN and SEGAs were very similar (see Fig. 1A-D, Fig. 2A-D). They were composed of spindled, epithelioid and gemistocyte-
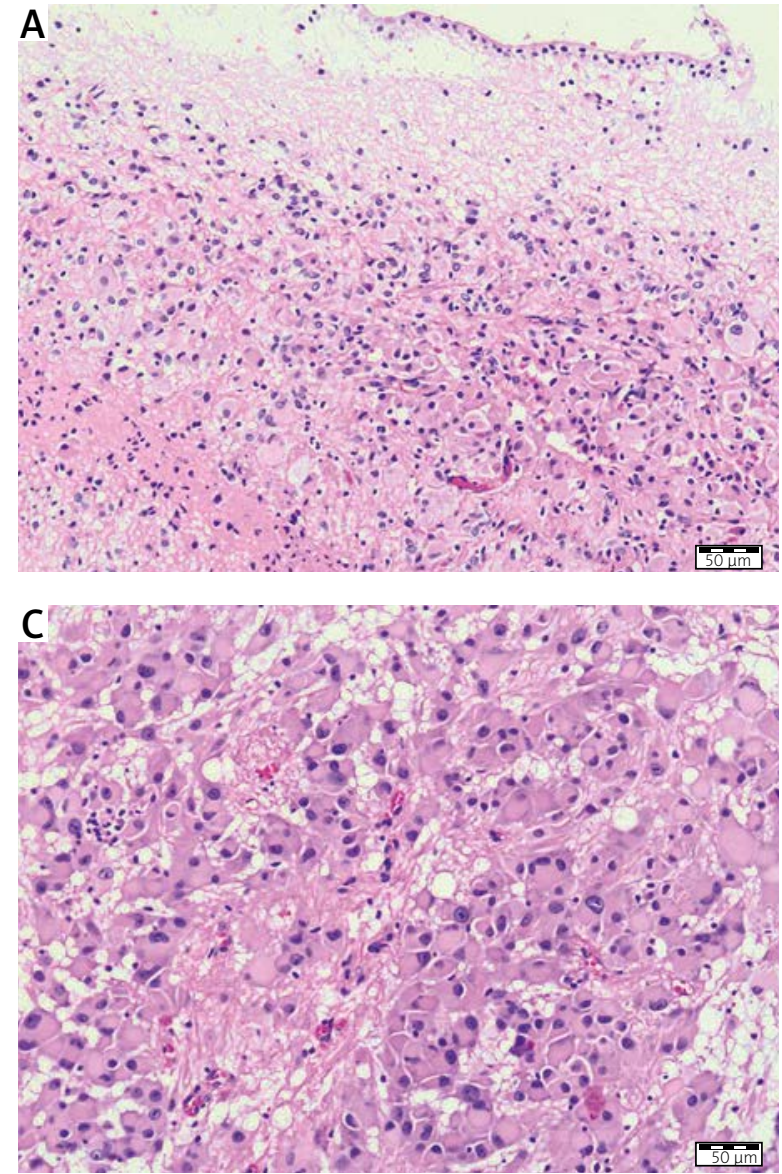

Fig. 2. Subependymal giant cell astrocytoma. A) Neoplastic cells covered by ependyma. B) Perivascular pseudorosettes. C) Gemistocytic-like cells in fibrillary background. D) Large, polygonal cells with abundant glassy cytoplasm and peripherally displaced nuclei. H\&E staining.

like cells and gliovascular stroma. The SEN was covered by a layer of ependymal cells (Fig. 1A). In addition, the largest cells with abundant glassy cytoplasm were situated in the central portions and smaller cells at the periphery of the SEN (Fig. 1B and C). Subependymal nodule displayed several calcifications (Fig. 1D). No mitotic figures or necrosis were detected in either SEN or SEGAs.

\section{Western blot results}

In both types of samples (SEN and SEGA) we found increased activation of S6rp, a protein involved in translation and cell proliferation. Cyclin D1, a G1/S cell cycle phase transition regulator, appeared to be markedly increased in both types of tissues. Raptor protein (regulatory-associated protein of mTOR), interestingly, was expressed only in the SEN specimen (Fig. 3A).
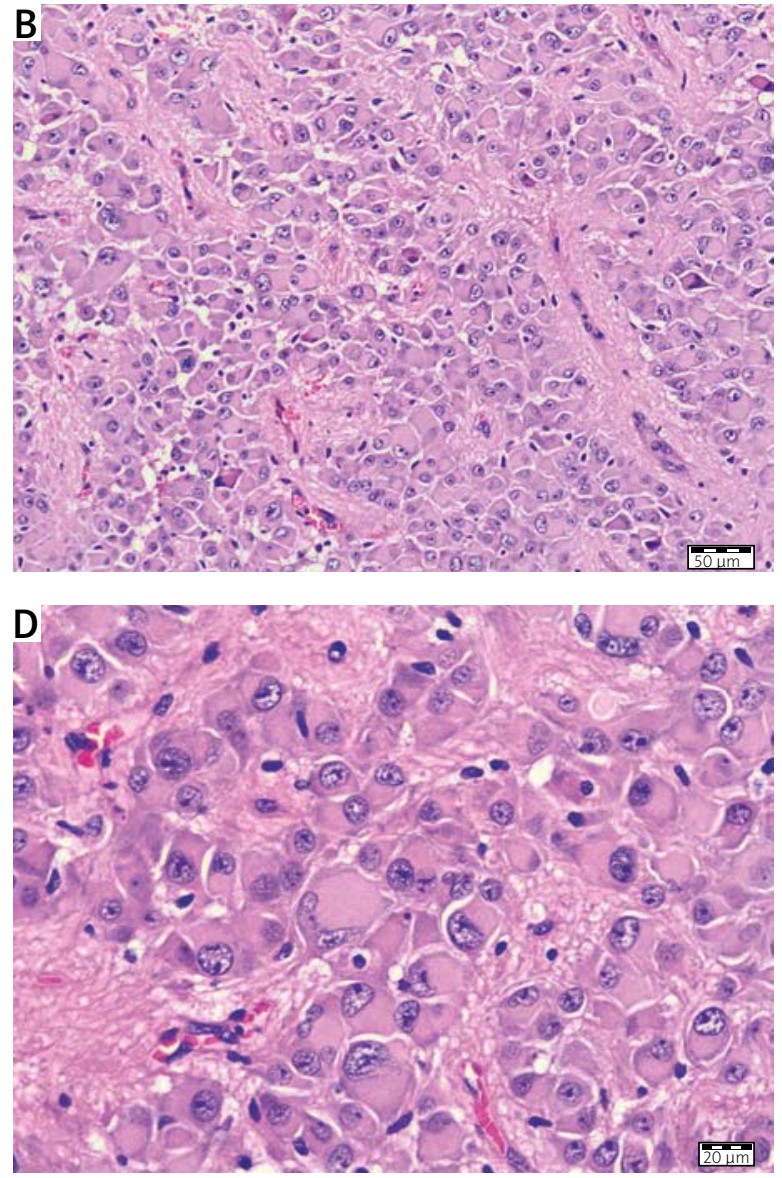
Then, we focused on three proteins taking part in the Akt cascade: PDK1, Akt and GSK3 $\beta$. We found that $p$-Akt, $p$-GSK3 $\beta$ (its effector) and p-PDK1, acting upstream of Akt, were upregulated in both SEN and SEGA from the same TS patient. The level of activation was similar in each sample and always significantly higher than in the control. Here, for the first time, we show Akt pathway activation in SEN (Fig. 3B).

As far as the Erk pathway is concerned, the results we obtained differ markedly for two types of lesions. Whilst there was no activation of p-Erk, p-Mek or
p-RSK1 in the SEN specimen, these proteins were significantly uptriggered in SEGA samples. The level of total Erk in SEGA and SEN samples was increased, compared to the control.

Also phosphorylated forms of stress kinases SAPK/ JNK (stress-activated protein kinase/c-Jun N-terminal kinases), other kinases from the Map kinase family, in both SEN and SEGA, were upregulated (Fig. 4).

We examined Bad and Bax proteins as well as caspase 9. Evaluation of these proteins, belonging to the apoptotic pathway, showed overactivation in
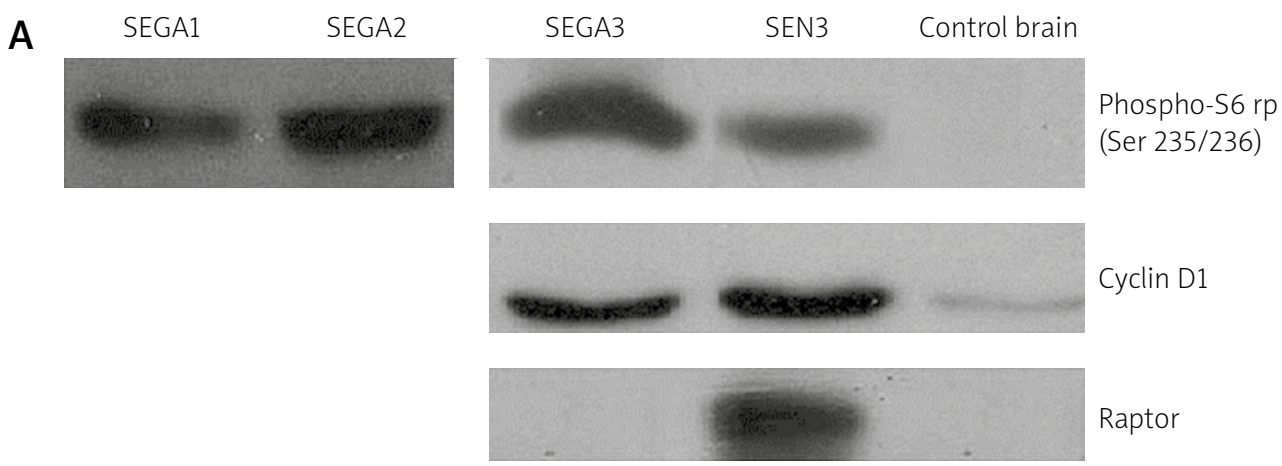

B
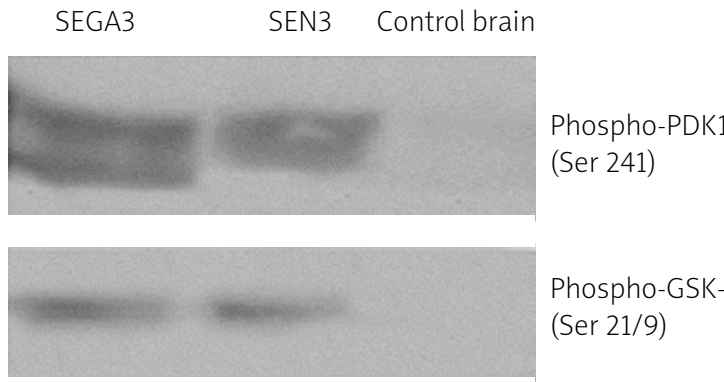

Phospho-GSK-3 beta (Ser 21/9)

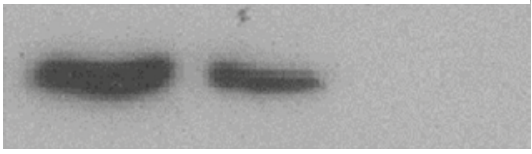

Phospho-Akt (Ser 473)

C
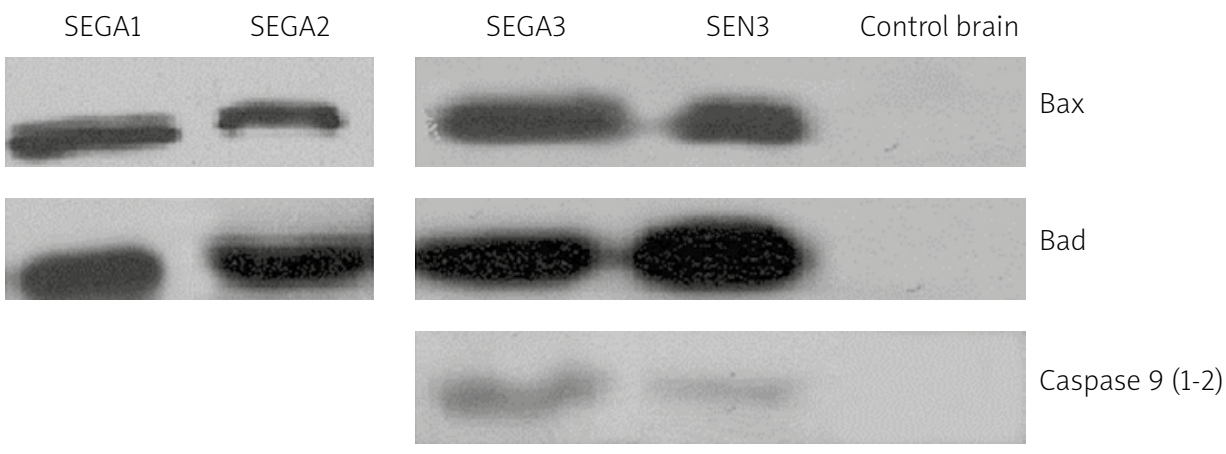

Fig. 3. Similarity of tumorigenic and apoptotic pathways in subependymal nodule (SEN) and subependymal giant cell astrocytoma (SEGA). A) Activation of mTOR effector, S6rp, cyclin D1 and raptor. B) Activation of Akt pathway. C) Activation of apoptosis. 


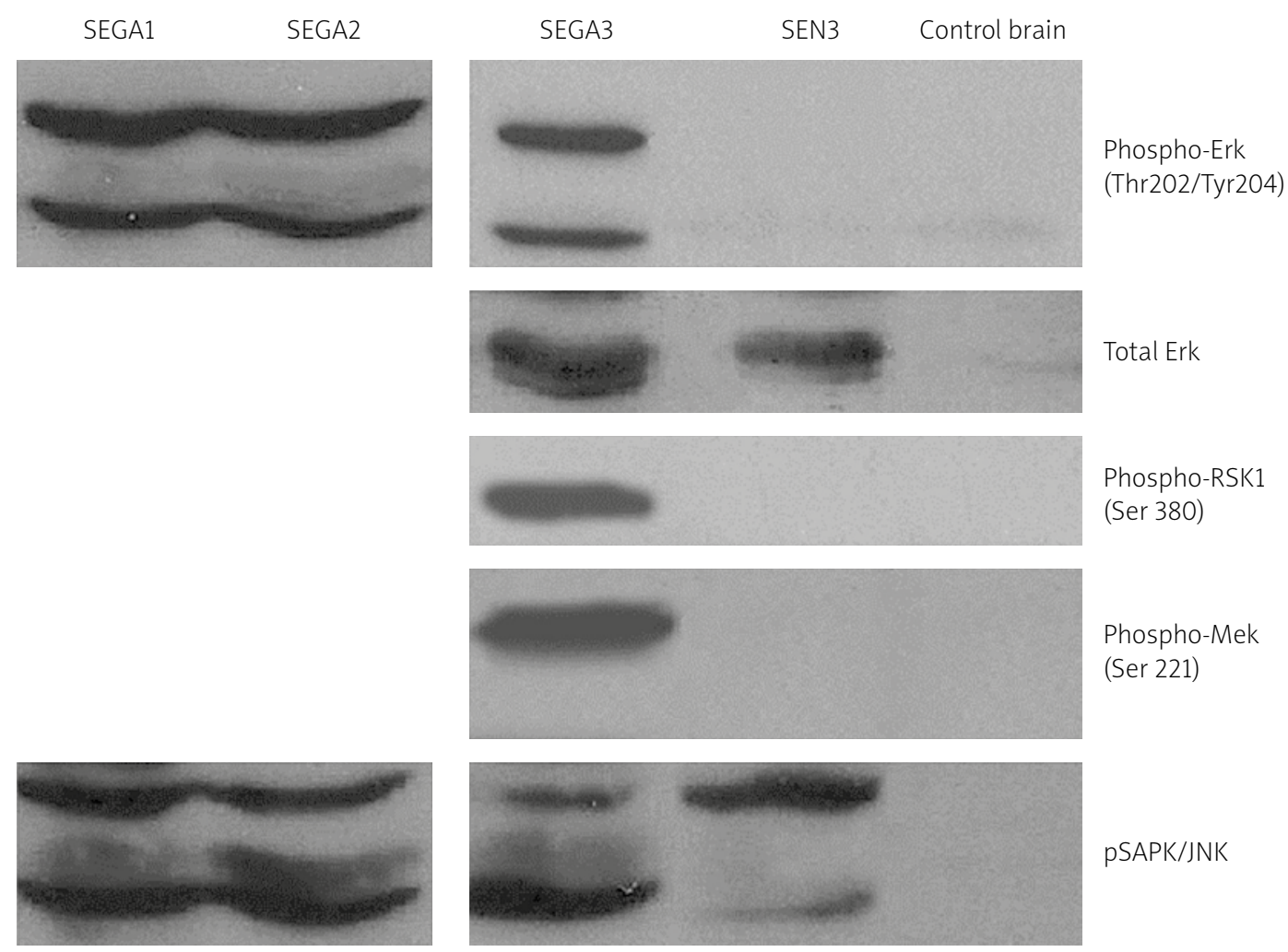

Fig. 4. Distinctive activation of Erk pathway in subependymal nodule (SEN), probably constituting the major difference between SEN and subependymal giant cell astrocytoma (SEGA).

both SEN and SEGA specimens, pointing to apoptosis activation (Fig. 3C).

\section{Conclusions and discussion}

Tumorigenesis is a complex phenomenon, involving disturbances of numerous signaling pathways: growth factor stimulation, cellular proliferation, apoptosis and cell death, DNA control, etc. The mammalian target of rapamycin pathway is located at the center of these events. Due to the fact that mTOR gathers signals associated with cellular well-being, such as the level of oxygen, nutrient availability or energy reserves, inhibiting cell proliferation if deficiency of any of these is detected, it is often a target of neoplastic transformation. Disturbances of pathways converging on mTOR are found in many epithelial tumors, including many CNS tumors of childhood $[8,13]$.

Akt participation in mTOR pathway activation in TS has been quite well recognized so far. The effect of Erk on mTOR and, generally, its role in TS pathogenesis are still going through the stage of exploration. In general, the studies are performed on a few samples of SEGAs $[5,6]$. According to our knowledge, there are no previous investigations on SEN samples involving Akt, Erk or mTOR pathways.

In our earlier study [6] we observed both Akt and Erk hyperactivation in SEGA specimens. We also found that the Erk pathway plays a more important role in tumor formation in TS than Akt, as Erk hyperactivation without Akt was sufficient for tumor progression. The results of our current study appear to confirm this observation.

As we know, no single event results in tumorigenesis. Our current study sheds some light on the possible mechanism of SEN/SEGA transformation, for which Erk pathway hyperactivation in SEGA may be significant. Our study shows that activation of the "classical" Erk kinase cascade leading to MTOR activation through Mek and RSK1 is different in SEN and SEGA. Also, transcription upregulation, leading to cell growth, differentiation, survival or promotion of apoptosis by overactivation of the stress-activated protein kinases (SAPK)/Jun amino-terminal kinases (JNK), was observed. Although it is too early to demonstrate the exact mechanism leading to 
SEGA appearance, one could suspect the following sequence of events: activation of PI3K/Akt, upregulation of mTOR (these two events leading to SEN formation, but too weak for the transformation of the cell into SEGA), and Erk activation (and thus appearance of SEGA). In view of the fact that SEN availability from human tissues is very scant, confirmation of this hypothesis may need some time. However, it may contribute to better future therapy of SEGA, as Erk inhibitors are already available on the market.

\section{Disclosure}

\section{Authors report no conflict of interest.}

\section{References}

1. Buccoliero AM, Franchi A, Castiglione F, Gheri CF, Mussa F, Giordano F, Gentori L, Taddei GL. Subependymal giant cell astrocytoma (SEGA): Is it an astrocytoma? Morphological, immunohistochemical and ultrastructural study. Neuropathology 2009; 29: 25-30.

2. Crino PB, Nathanson KL, Henske EP. The tuberous sclerosis complex. N Engl I Med 2006; 355: 1345-1356.

3. Grajkowska W, Kotulska K, Jurkiewicz E, Roszkowski M, Daszkiewicz P, Jóźwiak S, Matyja E. Subependymal giant cell astrocytomas with atypical histological features mimicking malignant gliomas. Folia Neuropathol 2011; 49: 39-46.

4. Gyure KA, Prayson RA. Subependymal giant cell astrocytoma: a clinicopathologic study with HMB45 and MIB-1 immunohistochemical analysis. Mod Pathol 1997; 10: 313-317.

5. Han S, Santos TM, Puga A, Roy J, Thiele EA, McCollin M, Stemmer-Rachamimov A, Ramesh V. Phosphorylation of tuberin as a novel mechanism for somatic inactivation of the tuberous sclerosis complex proteins in brain lesions. Cancer Res 2004; 64: 812-816.

6. Jozwiak J, Grajkowska W, Kotulska K, Jozwiak S, Zalewski W, Zajączkowska A, Roszkowski M, Slupianek A, Wlodarski P. Brain tumor formation in tuberous sclerosis depends on Erk activation. Neuromolecular Med 2007; 9: 117-127.

7. Jozwiak J, Jozwiak S, Wlodarski P. Possible mechanisms of disease development in tuberous sclerosis. Lancet Oncol 2008; 9: 73-79.

8. Jóźwiak J, Sontowska I, Bikowska B, Grajkowska W, Galus R, Roszkowski M. Favourable prognosis in medulloblastoma with extensive nodularity is associated with mitogen-activated protein kinase upregulation. Folia Neuropathol 2011; 49: 257-261.

9. Kalantari BN, Salamon N. Neuroimaging of tuberous sclerosis: spectrum of pathologic findings and frontiers in imaging. Am J Roentgenol 2008; 190: W304-W309.

10. Mizuguchi M, Takashima S. Neuropathology of tuberous sclerosis. Brain Dev 2001; 23: 508-515.

11. Northrup H, Krueger DA; International Tuberous Sclerosis Complex Consensus Group. Tuberous sclerosis complex diagnostic criteria update: recommendations of the 2012 International
Tuberous Sclerosis Complex Consensus Conference. Pediatr Neurol 2013; 49: 243-254.

12. Osborne JP, Fryer A, Webb D. Epidemiology of tuberous sclerosis. Ann N Y Acad Sci 1991; 615: 125-127.

13. Rak B, Szlufik S, Grajkowska W, Perek D, Dembowska-Bagińska B, Filipek I, Daszkiewicz P, Włodarski P, Jóźwiak J. Upregulation of mitogen-activated protein kinase in ganglioglioma. Folia Neuropathol 2013; 51: 283-289.

14. Roach ES, Gomez MR, Northrup H. Tuberous sclerosis complex consensus conference: revised clinical diagnostic criteria. J Child Neurol 1998; 13: 624-628.

15. Roth J, Roach ES, Bartels U, Jóźwiak S, Koenig MK, Weiner HL, Franz DN, Wang HZ. Subependymal giant cell astrocytoma: diagnosis, screening, and treatment. Recommendations from the International Tuberous Sclerosis Complex Consensus Conference 2012. Pediatr Neurol 2013; 49: 439-444.

16. Rovira A, Ruiz-Falco ML, Garcia-Esparza E, Lopez-Laso E, Macaya A, Malaga I, Vazquez E, Vincente J. Recommendations for the radiological diagnosis and follow-up of neuropathological abnormalities associated with tuberous sclerosis complex. J Neurooncol 2014; 118: 205-223.

17. Włodarski P, Grajkowska W, Łojek M, Rainko K, Jóźwiak J. Activation of Akt and Erk pathways in medulloblastoma. Folia Neuropathol 2006; 44: 214-220. 